CARPATHIAN J. MATH.

Volume 37 (2021), No. 2,

Pages 325 - 338
Online version at https : //www . carpathian. cunbm . utcluj. ro/

Print Edition: ISSN 1584 - 2851; Online Edition: ISSN 1843 - 4401

DOI: https://doi.org/10.37193/CJM.2021.02.17

Dedicated to Prof. Ioan A. Rus on the occasion of his $85^{\text {th }}$ anniversary

\title{
Positive solutions for second-order differential equations of Kirchhoff type on the half-line
}

\author{
Habiba Boulaiki, Toufik Moussaoui and Radu Precup
}

\begin{abstract}
The aim of the present paper is to study the existence of nontrivial nonnegative solutions for a second-order boundary value problem of Kirchhoff type on the half-line. Our approach is based on variational methods, a monotonicity trick related to the mountain pass lemma, cut-off functional technique, and a Pohozaev type identity.
\end{abstract}

\section{INTRODUCTION}

In this work, we are concerned with nontrivial nonnegative solutions to the following problem

$$
\left\{\begin{array}{l}
\left(a+\lambda \int_{0}^{+\infty}\left(u^{\prime}(t)^{2}+b u(t)^{2}\right) \mathrm{d} t\right)\left(-u^{\prime \prime}(t)+b u(t)\right)=f(u(t)) \text { for a.e. } t \in(0,+\infty) \\
u(0)=u(+\infty)=0
\end{array}\right.
$$

where $a$ and $b$ are positive constants, $\lambda \geq 0$ is a parameter and $f \in C\left(\mathbb{R}^{+}, \mathbb{R}^{+}\right)$.

We assume that the following conditions are satisfied:

(H1): There exists $\theta>1$ and nonnegative constants $\alpha, \beta$ such that $f(\xi) \leq \alpha+\beta \xi^{\theta}$ for all $\xi \in \mathbb{R}^{+}$.

(H2): $\lim _{\xi \rightarrow 0} \frac{f(\xi)}{\xi}=0$.

(H3): $\lim _{\xi \rightarrow+\infty} \frac{f(\xi)}{\xi}=+\infty$.

Problem (1.1) is a nonlocal one due to the presence of the term $\int_{0}^{+\infty}\left(u^{\prime 2}+b u^{2}\right) \mathrm{d} t$. This causes some mathematical difficulties which make its study particularly interesting.

This problem is related to the stationary Kirchhoff equation (see [14], [5] and [8]) describing the motion of a semi-infinite string

$$
\frac{\partial^{2} u}{\partial t^{2}}-\left(m_{0}+\int_{0}^{+\infty}\left(\frac{\partial u}{\partial x}\right)^{2} \mathrm{~d} x\right) \frac{\partial^{2} u}{\partial x^{2}}=h(t, x),
$$

where $m_{0}$ is a positive constant, $h(t, x)$ is the external force and $u(t, x)$ is the lateral displacement at the space coordinate $x$ and time $t$. This equation is an extension of the classical D'Alembert's wave equation and takes into account the length changes of the string produced by transverse vibrations. Notice that nonlocal problems also appear in other fields

Received: 28.04.2021. In revised form: 02.06.2021. Accepted: 03.06.2021

2010 Mathematics Subject Classification. 34B40, 47J30, 58E30.

Key words and phrases. Kirchhoff-type equation, equations on the half-line, variational method, mountain pass geometry, cut-off function technique, Pohozaev type identity.

Corresponding author: Radu Precup; r.precup@math.ubbcluj.ro 
such as biological systems, where, for example, $u$ is a population density whose dynamics depends on the average of the density itself.

If we set $a=1$ and $\lambda=0$, then (1.1) reduces to the following second order semi-linear boundary value problem on the half-line

$$
\left\{\begin{array}{l}
-u^{\prime \prime}(t)+b u(t)=f(u(t)) \text { for a.e. } t \in(0,+\infty) \\
u(0)=u(+\infty)=0 .
\end{array}\right.
$$

This type of problems has been extensively studied in the literature by several authors using different methods, for example, Zima [20] considered the following problem

$$
\left\{\begin{array}{l}
-u^{\prime \prime}(t)+k^{2} u(t)=f(t, u(t)), t \in(0,+\infty) \\
u(0)=u(+\infty)=0
\end{array}\right.
$$

where $k>0$ and $f$ is continuous and non-negative. By using a fixed point theorem in cones she proved the existence of at least one positive solution. The same problem has been studied in [2] by using variational methods and critical point theory in conical shells, where the authors established the existence and localization of at least two positive solutions. Also Ma and Zhu [17] by employing fixed-point theorems on cones, obtained the existence of positive solutions for the following second-order boundary value problem on the half-line

$$
\left\{\begin{array}{l}
-u^{\prime \prime}(t)+k^{2}(t) u(t)+\lambda m(t) f(t, u(t)), t \in(0,+\infty) \\
u(0)=u(+\infty)=0
\end{array}\right.
$$

where $k:[0, \infty) \rightarrow(0, \infty)$ and $f:[0, \infty) \times[0, \infty) \rightarrow \mathbb{R}$ are continuous. Gomes and Sanchez [7], by using variational methods and critical point theory proved the existence of solutions to the following boundary value problem

$$
\left\{\begin{array}{l}
-u^{\prime \prime}(t)+p(t) u(t)=\lambda q(t) f(u(t)) \text { for a.e. } t \in(0,+\infty) \\
u(0)=\alpha, \quad u(+\infty)=0
\end{array}\right.
$$

with $\alpha>0$ and $p, q:[0,+\infty) \rightarrow \mathbb{R}$ continuous functions. Bonanno and O'Regan [1] by using critical point arguments established some results on the existence of multiple solutions for a second order boundary value problem on the half-line.

In recent years the existence and multiplicity of solutions for second order problems of Kirchhoff type have been studied by many authors, for example, in [18], employing the Yang index and critical groups, Perera and Zhang obtained the existence of a non trivial solution for the following second order boundary value problem of Kirchhoff type

$$
\left\{\begin{array}{l}
-\left(a+b \int_{\Omega}|\nabla u|^{2} d x\right)(\Delta u)=f(x, u) \text { in } \Omega \\
u=0 \text { on } \partial \Omega
\end{array}\right.
$$

where $\Omega$ is a smooth bounded domain in $\mathbb{R}^{N}, N=1,2$ or $3, a, b>0$ and $f$ is a Carathéodory function on $\Omega \times \mathbb{R}$. In [15] Li, Li and Shi using variational methods and cut-off functional techniques established the existence of at least one positive solution for the following second-order nonlocal elliptic problem

$$
\left(a+\lambda \int_{\mathbb{R}^{N}}|\nabla u|^{2} d x+\lambda b \int_{\mathbb{R}^{N}} u^{2} d x\right)[-\Delta u+b u]=f(u) \quad \text { in } \mathbb{R}^{N},
$$

where $N \geq 3, a, b$ are positive constants and $\lambda \geq 0$ is a nonnegative parameter.

Also in [6], the authors using variational methods and iterative techniques studied the existence of non trivial positive solutions for problem (1.3) with $f(u)=K(x)|u|^{p-1} u$, $p \in(2,5)$ and $N=3$.

To the best of our knowledge, there are few papers considering Kirchhoff type problems on the half line except [4,9], where the authors studied second order boundary 
value problems of Kirchhoff type on the half-line with impulses by using variational methods and critical point theory.

Inspired by $[15,6]$, in the present paper we study the existence of at least one nontrivial nonnegative solution of problem(1.1) without the Ambrosetti-Rabinowitz (AR) condition on nonlinearity $f$. We use a cut-off function technique, a monotonicity trick related to the mountain pass lemma and a Pohozaev type identity, to obtain bounded Palais-Smale sequences.

The paper is organized as follows: In Section 2 we give some notations, preliminary concepts and results. Section 3 is devoted to proving our main result, namely the following theorem.

Theorem 1.1. Assume that conditions (H1), (H2) and (H3) hold. Then there exists $\lambda_{0}>0$ such that for any $\lambda \in\left[0, \lambda_{0}\right)$, problem (1.1) has at least one nonzero nonnegative solution.

Notice that since the result in Theorem 1.1 holds in particular for $\lambda=0$, under conditions (H1), (H2) and (H3), we obtain that problem (1.2) has a nonzero nonnegative solution.

\section{PRELIMINARIES}

Denote $\mathbb{R}^{+}=[0,+\infty)$ and by $\|u\|_{q}(q \geq 1)$ the $L^{q}\left(\mathbb{R}^{+}\right)$-norm. We consider the Sobolev space $H_{0}^{1}\left(\mathbb{R}^{+}\right)=\left\{u \in L^{2}\left(\mathbb{R}^{+}\right): u^{\prime} \in L^{2}\left(\mathbb{R}^{+}\right), u(0)=0\right\}$, endowed with inner product and norm

$$
(u, v)=\int_{0}^{+\infty}\left(u^{\prime}(t) v^{\prime}(t)+b u(t) v(t)\right) \mathrm{d} t, \quad\|u\|=\left(\int_{0}^{+\infty}\left(u^{\prime}(t)^{2}+b u(t)^{2}\right) \mathrm{d} t\right)^{1 / 2},
$$

we denote by $H^{-1}\left(\mathbb{R}^{+}\right)$the dual of $H_{0}^{1}\left(\mathbb{R}^{+}\right)$and we use the symbol $\langle\cdot, \cdot\rangle$ for the duality mapping between $H_{0}^{1}\left(\mathbb{R}^{+}\right)$and $H^{-1}\left(\mathbb{R}^{+}\right)$.

It is clear that the embedding $H_{0}^{1}\left(\mathbb{R}^{+}\right) \hookrightarrow L^{2}\left(\mathbb{R}^{+}\right)$is continuous and $\|u\|_{2} \leq \frac{1}{\sqrt{b}}\|u\|$, $u \in H_{0}^{1}\left(\mathbb{R}^{+}\right)$. Notice that if $u \in H_{0}^{1}\left(\mathbb{R}^{+}\right)$, then $u(+\infty)=0$ (see [3, Corollary 8.9]).

Since, contrary to the case of bounded intervals, the Sobolev embedding $H_{0}^{1}\left(\mathbb{R}^{+}\right) \hookrightarrow$ $C\left(\mathbb{R}^{+}\right)$is not compact, it is usually difficult to prove that a bounded Palais-Smale sequence has a strongly convergent subsequence, if we seek solutions of (1.1) by variational methods. To overcome this difficulty we consider a continuous function $p: \mathbb{R}^{+} \longrightarrow(0,+\infty)$ with $p^{-(\theta+1)} \in L^{1}\left(\mathbb{R}^{+}\right)$and

$$
\lim _{t \rightarrow+\infty} \sqrt{t} p(t)=0
$$

and the weighted Banach space $C_{l, p}\left(\mathbb{R}^{+}\right)$defined by

$$
C_{l, p}\left(\mathbb{R}^{+}\right)=\left\{u \in C\left(\mathbb{R}^{+}\right): \lim _{t \rightarrow+\infty} p(t) u(t)=0\right\},
$$

endowed with the norm $\|u\|_{\infty, p}=\sup _{t \in \mathbb{R}^{+}} p(t)|u(t)|$. Concerning this space we have the following result proved in [2].

Lemma 2.1. Assume that (2.4) holds. Then

(a): $H_{0}^{1}\left(\mathbb{R}^{+}\right)$is continuously embedded in $C_{l, p}\left(\mathbb{R}^{+}\right)$, more precisely, for every $u \in H_{0}^{1}\left(\mathbb{R}^{+}\right)$, one has

$$
\|u\|_{\infty, p} \leq c_{\infty, p}\left(\int_{0}^{+\infty} u^{\prime}(t)^{2} \mathrm{~d} t\right)^{1 / 2} \leq c_{\infty, p}\|u\|
$$

where $c_{\infty, p}=\sup _{t \in \mathbb{R}^{+}} \sqrt{t} p(t)$.

(b): The embedding $H_{0}^{1}\left(\mathbb{R}^{+}\right) \hookrightarrow C_{l, p}\left(\mathbb{R}^{+}\right)$is compact. 
Let $\widetilde{f}$ be the odd extension of $f$ to the whole $\mathbb{R}$, i.e., $\widetilde{f}(\xi)=-f(-\xi)$ for $\xi<0$,and $\widetilde{F}(\xi)=\int_{0}^{\xi} \widetilde{f}(s) \mathrm{d} s$. Note that from (H2), one has $f(0)=0$, and consequently the extension $\tilde{f}$ is continuous on $\mathbb{R}$.

We associate to problem (1.1) the energy functional $J_{\lambda}: H_{0}^{1}\left(\mathbb{R}^{+}\right) \rightarrow \mathbb{R}$,

$$
J_{\lambda}(u)=\frac{a}{2}\|u\|^{2}+\frac{\lambda}{4}\|u\|^{4}-\int_{0}^{+\infty} \widetilde{F}(u(t)) \mathrm{d} t .
$$

Under the assumptions on $f$ we have the following result.

Lemma 2.2. The energy functional $J_{\lambda}$ is of class $C^{1}$ on $H_{0}^{1}\left(\mathbb{R}^{+}\right)$and for every $u, v \in H_{0}^{1}\left(\mathbb{R}^{+}\right)$ one has

$$
\left\langle J_{\lambda}^{\prime}(u), v\right\rangle=\left(a+\lambda\|u\|^{2}\right)(u, v)-\int_{0}^{+\infty} \widetilde{f}(u(t)) v(t) \mathrm{d} t .
$$

Proof. Step 1: The functional $J_{\lambda}$ is well-defined. Indeed, for $u \in H_{0}^{1}\left(\mathbb{R}^{+}\right)$, the condition (H1) implies that

$$
|\widetilde{F}(u(t))| \leq\left|\int_{0}^{u(t)}\right| \widetilde{f}(\xi)|\mathrm{d} \xi| \leq\left|\int_{0}^{u(t)}\left(\alpha+\beta|\xi|^{\theta}\right) \mathrm{d} \xi\right| \leq \alpha|u(t)|+\frac{\beta}{\theta+1}|u(t)|^{\theta+1} .
$$

Then

$$
\begin{aligned}
\left|\int_{0}^{+\infty} \widetilde{F}(u(t)) \mathrm{d} t\right| & \leq \int_{0}^{+\infty}\left(\alpha|u(t)|+\frac{\beta}{\theta+1}|u(t)|^{\theta+1}\right) \mathrm{d} t \\
& =\alpha \int_{0}^{+\infty} \frac{1}{p(t)} p(t)|u(t)| \mathrm{d} t+\frac{\beta}{\theta+1} \int_{0}^{+\infty} \frac{1}{p^{\theta+1}(t)} p(t)^{\theta+1}|u(t)|^{\theta+1} \mathrm{~d} t \\
& \leq \alpha\|u\|_{\infty, p}\left\|p^{-1}\right\|_{1}+\frac{\beta}{\theta+1}\|u\|_{\infty, p}^{\theta+1}\left\|p^{-(\theta+1)}\right\|_{1} .
\end{aligned}
$$

Since $H_{0}^{1}\left(\mathbb{R}^{+}\right)$embeds continuously in $C_{l, p}\left(\mathbb{R}^{+}\right)$, then

$$
\left|\int_{0}^{+\infty} \widetilde{F}(u(t)) \mathrm{d} t\right| \leq\left(\alpha c_{\infty, p}\left\|p^{-1}\right\|_{1}+\frac{\beta c_{\infty, p}^{\theta+1}}{\theta+1}\left\|p^{-(\theta+1)}\right\|_{1}\|u\|^{\theta}\right)\|u\| .
$$

This shows that $J_{\lambda}$ is well-defined.

Step 2: The functional $J_{\lambda}$ is Gâteaux differentiable on $H_{0}^{1}\left(\mathbb{R}^{+}\right)$. Obviously, it suffices to prove this only for the functional

$$
\Phi(u):=\int_{0}^{+\infty} \widetilde{F}(u(t)) \mathrm{d} t .
$$

More exactly we prove that for any $u, v \in H_{0}^{1}\left(\mathbb{R}^{+}\right)$,

$$
\lim _{\tau \rightarrow 0} \frac{1}{\tau}(\Phi(u+\tau v)-\Phi(u))=\int_{0}^{+\infty} \widetilde{f}(u(t)) v(t) \mathrm{d} t,
$$

or equivalently

$$
\lim _{\tau \rightarrow 0} \int_{0}^{+\infty}\left(\frac{1}{\tau}(\widetilde{F}(u(t)+\tau v(t))-\widetilde{F}(u(t)))-\widetilde{f}(u(t)) v(t)\right) \mathrm{d} t=0,
$$

which shows that

$$
\left\langle\Phi^{\prime}(u), v\right\rangle=\int_{0}^{+\infty} \tilde{f}(u(t)) v(t) \mathrm{d} t .
$$


First, since $\widetilde{F}$ is a primitive of $\widetilde{f}$, then for every $t \in \mathbb{R}^{+}$, one has

$$
\frac{1}{\tau}(\widetilde{F}(u(t)+\tau v(t))-\widetilde{F}(u(t)))-\widetilde{f}(u(t)) v(t) \rightarrow 0 \quad \text { as } \tau \rightarrow 0 .
$$

Secondly, by the mean value theorem, for each $t \in \mathbb{R}^{+}$and $0<|\tau|<1$, there exists a number $\eta(t, \tau)$ with $0 \leq \eta(t, \tau) \leq 1$ such that

$$
\begin{aligned}
& \frac{1}{\tau}(\widetilde{F}(u(t)+\tau v(t))-\widetilde{F}(u(t)))-\widetilde{f}(u(t)) v(t) \\
= & (\tilde{f}(u(t)+\tau \eta(t, \tau) v(t))-\tilde{f}(u(t))) v(t) .
\end{aligned}
$$

Then

$$
\begin{aligned}
& \left|\frac{1}{\tau}(\widetilde{F}(u(t)+\tau v(t))-\widetilde{F}(u(t)))-\widetilde{f}(u(t)) v(t)\right| \\
\leq & |\widetilde{f}(u(t)+\tau \eta(t, \tau) v(t)) v(t)|+|\widetilde{f}(u(t)) v(t)| .
\end{aligned}
$$

By using the growth condition (H1) again, the inequality $(x+y)^{\theta} \leq 2^{\theta-1}\left(x^{\theta}+y^{\theta}\right)$ $\left(x, y \in \mathbb{R}^{+}, \theta \geq 1\right)$ and $0 \leq \eta(t, \tau) \leq 1$, we have

$$
\begin{aligned}
|\tilde{f}(u(t)+\tau \eta(t) v(t)) v(t)| & \leq\left(\alpha+\beta|u(t)+\tau \eta(t) v(t)|^{\theta}\right)|v(t)| \\
& \leq\left(\alpha+2^{\theta-1} \beta\left(|u(t)|^{\theta}+|\tau \eta(t) v(t)|^{\theta}\right)\right)|v(t)| \\
& \leq \alpha|v(t)|+2^{\theta-1} \beta\left(|u(t)|^{\theta}|v(t)|+|v(t)|^{\theta+1}\right) \\
& \leq \frac{\alpha}{p(t)}\|v\|_{\infty, p}+2^{\theta-1} \frac{\beta}{p(t)^{\theta+1}}\left(\|u\|_{\infty, p}^{\theta}\|v\|_{\infty, p}+\|v\|_{\infty, p}^{\theta+1}\right) .
\end{aligned}
$$

Similarly

$$
|\tilde{f}(u(t)) v(t)| \leq \frac{\alpha}{p(t)}\|v\|_{\infty, p}+2^{\theta-1} \frac{\beta}{p(t)^{\theta+1}}\left(\|u\|_{\infty, p}^{\theta}\|v\|_{\infty, p}+\|v\|_{\infty, p}^{\theta+1}\right) .
$$

Since the embedding $H_{0}^{1}\left(\mathbb{R}^{+}\right) \hookrightarrow C_{l, p}\left(\mathbb{R}^{+}\right)$is continuous, (2.8), (2.9) and (2.10) yield

$$
\begin{aligned}
& \left|\frac{1}{\tau}(\widetilde{F}(u(t)+\tau v(t))-\widetilde{F}(u(t)))-\widetilde{f}(u(t)) v(t)\right| \\
\leq & 2\left(\frac{\alpha}{p(t)}\|v\|_{\infty, p}+2^{\theta-1} \frac{\beta}{p(t)^{\theta+1}}\left(\|u\|_{\infty, p}^{\theta}\|v\|_{\infty, p}+\|v\|_{\infty, p}^{\theta+1}\right)\right) \\
\leq & 2 \alpha c_{\infty, p}\|v\| \frac{1}{p(t)}+2^{\theta} \beta c_{\infty, p}^{\theta+1}\left(\|u\|^{\theta}\|v\|+\|v\|^{\theta+1}\right) \frac{1}{p(t)^{\theta+1}}=: g(t) .
\end{aligned}
$$

Since $p^{-(\theta+1)} \in L^{1}\left(\mathbb{R}^{+}\right)$, one also has $p^{-1} \in L^{1}\left(\mathbb{R}^{+}\right)$and then $g \in L^{1}\left(\mathbb{R}^{+}\right)$. So the Lebesgue dominated convergence theorem implies that (2.6) is true. Thus, $\Phi$ is Gâteaux differentiable and (2.7) holds.

Step 3. $J_{\lambda}^{\prime}$ is continuous. Here again, it suffices to prove this property for $\Phi^{\prime}$. Let $\left\{u_{n}\right\} \subset H_{0}^{1}\left(\mathbb{R}^{+}\right)$be such that $u_{n} \rightarrow u$ in $H_{0}^{1}\left(\mathbb{R}^{+}\right)$as $n \rightarrow+\infty$. Then $u_{n} \rightarrow u$ in $C_{l, p}\left(\mathbb{R}^{+}\right)$as $n \rightarrow+\infty$. So $u_{n}(t) \rightarrow u(t)$ for every $t \in \mathbb{R}^{+}$and there exists $r>0$ such that $\left\|u_{n}\right\|_{\infty, p} \leq r$ for all $n$. Thus, from the continuity of $\tilde{f}$, we have $\widetilde{f}\left(u_{n}(t)\right) \rightarrow \widetilde{f}(u(t))$ as $n \rightarrow+\infty$ for every $t \in \mathbb{R}^{+}$.

Furthermore, using (H1) and a similar reasoning to the above one, by the Lebesgue dominated convergence theorem, we have

$$
\int_{0}^{+\infty} \tilde{f}\left(u_{n}(t)\right) v(t) \mathrm{d} t \rightarrow \int_{0}^{+\infty} \tilde{f}(u(t)) v(t) \mathrm{d} t \quad \text { as } n \rightarrow+\infty
$$


uniformly with respect to $v \in H_{0}^{1}\left(\mathbb{R}^{+}\right)$with $\|v\| \leq 1$. As a consequence, $\| \Phi^{\prime}\left(u_{n}\right)-$ $\Phi^{\prime}(u) \|_{H^{-1}\left(\mathbb{R}^{+}\right)} \rightarrow 0$ as $n \rightarrow+\infty$, which proves the continuity of $\Phi^{\prime}$.

It is standard to verify that the critical points of the functional $J_{\lambda}$ are the weak solutions of the problem

$$
\left\{\begin{array}{l}
\left(a+\lambda \int_{0}^{+\infty}\left(u^{\prime}(t)^{2}+b u(t)^{2}\right) \mathrm{d} t\right)\left(-u^{\prime \prime}(t)+b u(t)\right)=\widetilde{f}(u(t)) \text { for a.e. } t \in(0,+\infty) \\
u(0)=u(+\infty)=0
\end{array}\right.
$$

and if $u$ solves (2.11), then the function $|u|$ is a nonnegative solution of (1.1).

The main tool for establishing our existence result is the following "monotonicity trick" theorem due to Struwe [19] and Jeanjean [10], [11].

Theorem $2.2([10,11])$. Let $\left(X,\|\cdot\|_{X}\right)$ be a Banach space and $I \subset \mathbb{R}_{+}$an interval. Consider a family of $C^{1}$ functionals on $X$ of the form

$$
J_{\mu}(u)=A(u)-\mu B(u), \quad \mu \in I,
$$

with $J_{\mu}(0)=0$ for every $\mu \in I, B$ nonnegative, and either $A(u) \rightarrow+\infty$ or $B(u) \rightarrow+\infty$ as $\|u\|_{X} \rightarrow+\infty$. For any $\mu \in I$, let

$$
\Gamma_{\mu}=\left\{\gamma \in C([0,1], X): \gamma(0)=0, J_{\mu}(\gamma(1))<0\right\} .
$$

If for each $\mu \in I$, the set $\Gamma_{\mu}$ is nonempty and

$$
c_{\mu}=\inf _{\gamma \in \Gamma_{\mu}} \max _{s \in[0,1]} J_{\mu}(\gamma(s))>0,
$$

then for almost every $\mu \in I$ there is a sequence $\left\{u_{n}\right\} \subset X$ such that

(i) $\left\{u_{n}\right\}$ is bounded;

(ii) $J_{\mu}\left(u_{n}\right) \rightarrow c_{\mu}$ as $n \rightarrow+\infty$;

(iii) $J_{\mu}^{\prime}\left(u_{n}\right) \rightarrow 0$ as $n \rightarrow+\infty$, in the dual $X^{-1}$ of $X$.

\section{PROOF OF THE MAIN RESUlT}

In this section, we use Theorem 2.2 to proof our main result. Since the nonlinearity $\tilde{f}$ may not satisfy the Ambrosetti-Rabinowitz condition, it is difficult to obtain the boundedness of any Palais-Smale sequence for the functional $J_{\lambda}$, even if a Palais-Smale sequence has been obtained. To overcome this difficulty, we adopt the cut-off functional technique introduced in $[12,13]$ (see also $[15,16])$. We use a cut-off function $\psi \in C^{1}\left(\mathbb{R}^{+},[0,1]\right)$ satisfying

$$
\begin{aligned}
& \psi(t)=1 \text { for } t \in[0,1], \quad 0 \leq \psi(t) \leq 1 \text { for } t \in(1,2), \\
& \psi(t)=0 \text { for } t \in[2, \infty), \quad\left\|\psi^{\prime}\right\|_{\infty} \leq 2,
\end{aligned}
$$

and for any $T>0$, we introduce the following truncated functional $J_{\lambda}^{T}: H_{0}^{1}\left(\mathbb{R}^{+}\right) \rightarrow \mathbb{R}$,

$$
J_{\lambda}^{T}(u)=\frac{a}{2}\|u\|^{2}+\frac{\lambda}{4} h_{T}(u)\|u\|^{4}-\int_{0}^{+\infty} \widetilde{F}(u(t)) \mathrm{d} t,
$$

where $h_{T}(u)=\psi\left(\frac{\|u\|^{2}}{T^{2}}\right)$. Of course, any critical point $u$ of $J_{\lambda}^{T}$ with $\|u\| \leq T$ is a critical point of $J_{\lambda}$. The number $T$ will be conveniently chosen later.

In order to apply Theorem 2.2, we take $X=H_{0}^{1}\left(\mathbb{R}^{+}\right), I=[\delta, 1]$ for some fixed $\delta \in(0,1)$, and

$$
A(u)=\frac{1}{2} a\|u\|^{2}+\frac{1}{4} \lambda h_{T}(u)\|u\|^{4}, \quad B(u)=\int_{0}^{+\infty} \widetilde{F}(u(t)) \mathrm{d} t .
$$


So for a given $\lambda \geq 0$, we consider the family of functionals depending on the parameter $\mu \in I$, namely

$$
J_{\lambda, \mu}^{T}(u)=\frac{a}{2}\|u\|^{2}+\frac{\lambda}{4} h_{T}(u)\|u\|^{4}-\mu \int_{0}^{+\infty} \widetilde{F}(u(t)) \mathrm{d} t, \quad u \in H_{0}^{1}\left(\mathbb{R}^{+}\right) .
$$

Since $\widetilde{F} \geq 0$ on $\mathbb{R}$, one has that $B$ is nonnegative on $H_{0}^{1}\left(\mathbb{R}^{+}\right)$. Also, it is clear that $A(u) \rightarrow$ $+\infty$ as $\|u\| \rightarrow+\infty$ and $J_{\lambda, \mu}^{T}(0)=0$. By a similar proof to that of Lemma 2.2, we can see that $J_{\lambda, \mu}^{T}$ is of class $C^{1}$ on $H_{0}^{1}\left(\mathbb{R}^{+}\right)$and for every $u, v \in H_{0}^{1}\left(\mathbb{R}^{+}\right)$,

$$
\begin{aligned}
\left\langle\left(J_{\lambda, \mu}^{T}\right)^{\prime}(u), v\right\rangle= & \left(a+\lambda h_{T}(u)\|u\|^{2}+\frac{\lambda}{2 T^{2}} \psi^{\prime}\left(\frac{\|u\|^{2}}{T^{2}}\right)\|u\|^{4}\right)(u, v) \\
& -\mu \int_{0}^{+\infty} \tilde{f}(u(t)) v(t) \mathrm{d} t .
\end{aligned}
$$

Next we show that the other assumptions of Theorem 2.2 are fulfilled by the functionals $J_{\lambda, \mu}^{T}$.

Denote

$$
\begin{gathered}
\Gamma_{\lambda, \mu}=\left\{\gamma \in C\left([0,1], H_{0}^{1}\left(\mathbb{R}^{+}\right)\right): \gamma(0)=0, J_{\lambda, \mu}^{T}(\gamma(1))<0\right\}, \\
c_{\lambda, \mu}=\inf _{\gamma \in \Gamma_{\lambda, \mu}} \max _{s \in[0,1]} J_{\lambda, \mu}^{T}(\gamma(s)) .
\end{gathered}
$$

Lemma 3.3. For every $\lambda \geq 0$ and $\mu \in I$, one has $\Gamma_{\lambda, \mu} \neq \emptyset$.

Proof. Let us consider a fixed $\phi \in C_{0}^{\infty}\left(\mathbb{R}^{+}\right)$with $\phi \geq 0,\|\phi\|=1$, and assume that $\operatorname{supp}(\phi) \subset[0, R]$ for some $R>0$. By (H3), we have that for any $M>0$ with

$$
M \delta \int_{0}^{R} \phi^{2}(t) d t>\frac{a}{2}
$$

there exists a constant $C(M)>0$ such that $\widetilde{F}(\xi) \geq M \xi^{2}-C(M)$ for all $\xi \in \mathbb{R}$. Then, for $s>0$ with $s^{2} \geq 2 T^{2}$, one has

$$
\begin{aligned}
J_{\lambda, \mu}^{T}(s \phi) & =\frac{a}{2} s^{2}\|\phi\|^{2}+\frac{\lambda}{4} \psi\left(\frac{s^{2}\|\phi\|^{2}}{T^{2}}\right) s^{4}\|\phi\|^{4}-\mu \int_{0}^{+\infty} \widetilde{F}(s \phi(t)) \mathrm{d} t \\
& =\frac{a}{2} s^{2}-\mu \int_{0}^{+\infty} \widetilde{F}(s \phi(t)) \mathrm{d} t \\
& \leq \frac{a}{2} s^{2}-\delta \int_{0}^{R}\left(M s^{2} \phi^{2}(t)-C(M)\right) \mathrm{d} t \\
& \leq\left(\frac{a}{2}-M \delta \int_{0}^{R} \phi^{2}(t) \mathrm{d} t\right) s^{2}+C(M) R \delta .
\end{aligned}
$$

Then in view of (3.13) we can choose $s_{0}>0$ large enough that $J_{\lambda, \mu}^{T}\left(s_{0} \phi\right)<0$. Consequently, the path $\gamma(s)=s s_{0} \phi \quad(s \in[0,1])$ belongs to $\Gamma_{\lambda, \mu}$ and thus $\Gamma_{\lambda, \mu} \neq \emptyset$.

Lemma 3.4. There exists a positive constant $c$ such that $c_{\lambda, \mu} \geq c$ for all $\lambda \geq 0$ and $\mu \in I$.

Proof. By (H1) and (H2), we see that for any $\epsilon>0$ (let it be $<a b / 2$ ), there exists a constant $C_{\epsilon}>0$ such that

$$
|\widetilde{f}(\xi)| \leq \epsilon|\xi|+C_{\epsilon}|\xi|^{\theta} \quad \text { for all } \xi \in \mathbb{R}
$$

Then

$$
|\widetilde{F}(\xi)| \leq \frac{\epsilon}{2} \xi^{2}+\frac{C_{\epsilon}}{\theta+1}|\xi|^{\theta+1} \quad \text { for all } \xi \in \mathbb{R}
$$


Hence for any $u \in H_{0}^{1}\left(\mathbb{R}^{+}\right)$, (3.15) implies

$$
\begin{aligned}
\int_{0}^{+\infty} \widetilde{F}(u(t)) \mathrm{d} t & \leq \int_{0}^{+\infty}\left(\frac{\epsilon}{2}|u(t)|^{2}+\frac{C_{\epsilon}}{\theta+1}|u(t)|^{\theta+1}\right) \mathrm{d} t \\
& \leq \frac{\epsilon}{2} \int_{0}^{+\infty}|u(t)|^{2} \mathrm{~d} t+\frac{C_{\epsilon}}{\theta+1} \int_{0}^{+\infty} \frac{1}{p(t)^{\theta+1}} p(t)^{\theta+1}|u(t)|^{\theta+1} \mathrm{~d} t \\
& \leq \frac{\epsilon}{2}\|u\|_{2}^{2}+\frac{C_{\epsilon}}{\theta+1}\left\|p^{-(\theta+1)}\right\|_{1}\|u\|_{\infty, p}^{\theta+1} .
\end{aligned}
$$

Furthermore, using the fact that $H_{0}^{1}\left(\mathbb{R}^{+}\right)$embeds continuously in $L^{2}\left(\mathbb{R}^{+}\right)$and in $C_{l, p}\left(\mathbb{R}^{+}\right)$ one has

$$
\int_{0}^{+\infty} \widetilde{F}(u(t)) \mathrm{d} t \leq \frac{\epsilon}{2 b}\|u\|^{2}+\frac{C_{\epsilon}}{\theta+1} c_{\infty, p}^{\theta+1}\left\|p^{-(\theta+1)}\right\|_{1}\|u\|^{\theta+1} .
$$

Then for every $\lambda \geq 0$ and $\mu \in I$, we have

$$
\begin{aligned}
J_{\lambda, \mu}^{T}(u) & =\frac{a}{2}\|u\|^{2}+\frac{\lambda}{4} h_{T}(u)\|u\|^{4}-\mu \int_{0}^{+\infty} \widetilde{F}(u(t)) \mathrm{d} t \\
& \geq \frac{a}{2}\|u\|^{2}-\int_{0}^{+\infty} \widetilde{F}(u(t)) \mathrm{d} t \\
& \geq \frac{a}{2}\|u\|^{2}-\frac{\epsilon}{2 b}\|u\|^{2}-\frac{C_{\epsilon}}{\theta+1} c_{\infty, p}^{\theta+1}\left\|p^{-(\theta+1)}\right\|_{1}\|u\|^{\theta+1} \\
& =\left[\left(\frac{a}{2}-\frac{\epsilon}{2 b}\right)-\frac{C_{\epsilon}}{\theta+1} c_{\infty, p}^{\theta+1}\left\|p^{-(\theta+1)}\right\|_{1}\|u\|^{\theta-1}\right]\|u\|^{2} .
\end{aligned}
$$

Since $\theta>1$, there exists $\rho>0$ such that if $\|u\| \leq \rho$, then

$$
\frac{C_{\epsilon}}{\theta+1} c_{\infty, p}^{\theta+1}\left\|p^{-(\theta+1)}\right\|_{1}\|u\|^{\theta-1} \leq \frac{a}{8} .
$$

Thus, since $\epsilon<\frac{a b}{2}$, one has $\frac{a}{2}-\frac{\epsilon}{2 b} \geq \frac{a}{4}$, and then for all $\lambda \geq 0, \mu \in I$ and $u \in H_{0}^{1}\left(\mathbb{R}^{+}\right)$ with $0<\|u\| \leq \rho$, we have

$$
J_{\lambda, \mu}^{T}(u) \geq \frac{a}{8}\|u\|^{2}
$$

In particular, for $\|u\|=\rho$, we have

$$
J_{\lambda, \mu}^{T}(u) \geq \frac{a}{8} \rho^{2}=: c>0 .
$$

Now fix $\lambda \geq 0$ and $\mu \in I$ and take any $\gamma \in \Gamma_{\lambda, \mu}$. Since by the definition of $\Gamma_{\lambda, \mu}$ one has $J_{\lambda, \mu}^{T}(\gamma(1))<0$, we must have $\|\gamma(1)\|>\rho$. Since $\gamma(0)=0$, then there exists $s_{\gamma} \in(0,1)$ such that $\left\|\gamma\left(s_{\gamma}\right)\right\|=\rho$. Therefore $J_{\lambda, \mu}^{T}\left(\gamma\left(s_{\gamma}\right)\right) \geq c$. Now

$$
c_{\lambda, \mu}=\inf _{\gamma \in \Gamma_{\lambda, \mu}} \max _{s \in[0,1]} J_{\lambda, \mu}^{T}(\gamma(s)) \geq \inf _{\gamma \in \Gamma_{\lambda, \mu}} J_{\lambda, \mu}^{T}\left(\gamma\left(s_{\gamma}\right)\right) \geq c
$$

which finishes the proof.

Next we prove that if $\lambda$ is small enough, then for any $\mu \in I$, every bounded PalaisSmale sequence of the functional $J_{\lambda, \mu}^{T}$ at level $c_{\lambda, \mu}$ has a convergent subsequence.

Lemma 3.5. Assume that $4 \lambda T^{2}<a$. Then for any $\mu \in I$, every bounded Palais-Smale sequence of $J_{\lambda, \mu}^{T}$ at level $c_{\lambda, \mu}$ has a convergent subsequence. 
Proof. Let $\mu \in I$ and let $\left\{v_{n}\right\}$ be a bounded Palais-Smale sequence of $J_{\lambda, \mu}^{T}$. Assume that $\left\|v_{n}\right\| \leq C$ for every $n$. Then

$$
\begin{gathered}
\left\{v_{n}\right\} \text { and }\left\{J_{\lambda, \mu}^{T}\left(v_{n}\right)\right\} \text { are bounded, } \\
\left(J_{\lambda, \mu}^{T}\right)^{\prime}\left(v_{n}\right) \rightarrow 0 \quad \text { in } H^{-1}\left(\mathbb{R}^{+}\right) \text {as } n \rightarrow+\infty .
\end{gathered}
$$

Since the embedding $H_{0}^{1}\left(\mathbb{R}^{+}\right) \hookrightarrow C_{l, p}\left(\mathbb{R}^{+}\right)$is compact (see Lemma $2.1(\mathrm{~b})$ ), up to a subsequence, there exists $v \in H_{0}^{1}\left(\mathbb{R}^{+}\right)$such that

$$
v_{n} \rightarrow v \quad \text { weakly in } H_{0}^{1}\left(\mathbb{R}^{+}\right) \text {and } v_{n} \rightarrow v \text { strongly in } C_{l, p}\left(\mathbb{R}^{+}\right) .
$$

Using (H1), the continuity of the embedding $H_{0}^{1}\left(\mathbb{R}^{+}\right) \hookrightarrow C_{l, p}\left(\mathbb{R}^{+}\right)$and the boundedness of $\left\{v_{n}\right\}$, we obtain

$$
\begin{aligned}
\left|\int_{0}^{+\infty} \widetilde{f}\left(v_{n}(t)\right)\left(v_{n}(t)-v(t)\right) \mathrm{d} t\right| & \leq \int_{0}^{+\infty} \widetilde{f}\left(v_{n}(t)\right)|| v_{n}(t)-v(t) \mid \mathrm{d} t \\
& \leq \int_{0}^{+\infty} \alpha\left|v_{n}(t)-v(t)\right| \mathrm{d} t+\beta \int_{0}^{+\infty}\left|v_{n}(t)\right|^{\theta}\left|v_{n}(t)-v(t)\right| \mathrm{d} t \\
& \leq \alpha\left\|p^{-1}\right\|_{1}\left\|v_{n}-v\right\|_{\infty, p}+\beta\left\|p^{-(\theta+1)}\right\|\left\|_{1}\right\| v_{n}\left\|_{\infty, p}^{\theta}\right\| v_{n}-v \|_{\infty, p} \\
& \leq \alpha\left\|p^{-1}\right\|_{1}\left\|v_{n}-v\right\|_{\infty, p}+\beta c_{\infty, p}^{\theta} C^{\theta}\left\|p^{-(\theta+1)}\right\|_{1}\left\|v_{n}-v\right\|_{\infty, p} .
\end{aligned}
$$

Then, by (3.16) we have

$$
\int_{0}^{+\infty} \widetilde{f}\left(v_{n}(t)\right)\left(v_{n}(t)-v(t)\right) \mathrm{d} t \rightarrow 0 \quad \text { as } n \rightarrow+\infty .
$$

Moreover, since $\left(J_{\lambda, \mu}^{T}\right)^{\prime}\left(v_{n}\right) \rightarrow 0$ and $v_{n} \rightarrow v$ weakly in $H_{0}^{1}\left(\mathbb{R}^{+}\right)$, one has

$$
\left\langle\left(J_{\lambda, \mu}^{T}\right)^{\prime}\left(v_{n}\right), v_{n}-v\right\rangle \rightarrow 0 \quad \text { as } n \rightarrow+\infty .
$$

Hence, using the expression of $\left(J_{\lambda, \mu}^{T}\right)^{\prime}$ we obtain

$$
\begin{aligned}
& {\left[a+\lambda h_{T}\left(v_{n}\right)\left\|v_{n}\right\|^{2}+\frac{\lambda}{2 T^{2}} \psi^{\prime}\left(\frac{\left\|v_{n}\right\|^{2}}{T^{2}}\right)\left\|v_{n}\right\|^{4}\right]\left(v_{n}, v_{n}-v\right)} \\
& =\left\langle\left(J_{\lambda, \mu}^{T}\right)^{\prime}\left(v_{n}\right), v_{n}-v\right\rangle+\mu \int_{0}^{+\infty} \widetilde{f}(v(t))\left(v_{n}(t)-v(t)\right) \mathrm{d} t \rightarrow 0 \quad \text { as } n \rightarrow+\infty .
\end{aligned}
$$

Now we show that

$$
\left(v_{n}, v_{n}-v\right) \rightarrow 0 \quad \text { as } n \rightarrow+\infty .
$$

If for some index $n$ one has $\left\|v_{n}\right\|^{2}>2 T^{2}$, then

$$
h_{T}\left(v_{n}\right)=0 \quad \text { and } \quad \psi^{\prime}\left(\frac{\left\|v_{n}\right\|^{2}}{T^{2}}\right)=0 .
$$

\section{Consequently}

$$
\left|a+\lambda h_{T}\left(v_{n}\right)\left\|v_{n}\right\|^{2}+\frac{\lambda}{2 T^{2}} \psi^{\prime}\left(\frac{\left\|v_{n}\right\|^{2}}{T^{2}}\right)\left\|v_{n}\right\|^{4}\right|=a .
$$

If for an index $n$ one has $\left\|v_{n}\right\|^{2} \leq 2 T^{2}$, then

$$
0 \leq h_{T}\left(v_{n}\right) \leq 1, \quad\left|\psi^{\prime}\left(\frac{\left\|v_{n}\right\|^{2}}{T^{2}}\right)\right| \leq\left\|\psi^{\prime}\right\|_{\infty} \leq 2,
$$


and so

$$
\left|\psi^{\prime}\left(\frac{\left\|v_{n}\right\|^{2}}{T^{2}}\right)\left\|v_{n}\right\|^{4}\right| \leq 8 T^{4} \quad \text { and } \quad 0 \leq h_{T}\left(v_{n}\right)\left\|v_{n}\right\|^{2} \leq 2 T^{2} .
$$

Then since $4 \lambda T^{2}<a$, we have

$$
\begin{aligned}
& \left|a+\lambda h_{T}\left(v_{n}\right)\left\|v_{n}\right\|^{2}+\frac{\lambda}{2 T^{2}} \psi^{\prime}\left(\frac{\left\|v_{n}\right\|^{2}}{T^{2}}\right)\left\|v_{n}\right\|^{4}\right| \\
\geq & a+\lambda h_{T}\left(v_{n}\right)\left\|v_{n}\right\|^{2}-\frac{\lambda}{2 T^{2}}\left|\psi^{\prime}\left(\frac{\left\|v_{n}\right\|^{2}}{T^{2}}\right)\left\|v_{n}\right\|^{4}\right| \\
\geq & a-\frac{\lambda}{2 T^{2}} 8 T^{4}=a-4 \lambda T^{2}>0 .
\end{aligned}
$$

Thus, in view of (3.20) and (3.22), if $4 \lambda T^{2}<a$, then for every $n$,

$$
\left|a+\lambda h_{T}\left(v_{n}\right)\left\|v_{n}\right\|^{2}+\frac{\lambda}{2 T^{2}} \psi^{\prime}\left(\frac{\left\|v_{n}\right\|^{2}}{T^{2}}\right)\left\|v_{n}\right\|^{4}\right| \geq a-4 \lambda T^{2}>0 .
$$

This together with (3.17) yields (3.18).

On the other hand, since $v_{n} \rightarrow v$ weakly in $H_{0}^{1}\left(\mathbb{R}^{+}\right)$, we have $\left(v, v_{n}-v\right) \rightarrow 0$. This together with (3.18) implies that $\left\|v_{n}-v\right\| \rightarrow 0$, that is $v_{n} \rightarrow v$ strongly in $H_{0}^{1}\left(\mathbb{R}^{+}\right)$, as desired.

Remark 3.1. (a) The discussion leading to (3.19) and (3.21) shows that for any $u \in H_{0}^{1}\left(R^{+}\right)$, one has

$$
h_{T}(u)\|u\|^{4} \leq 4 T^{4} \text { and }\left|\psi^{\prime}\left(\frac{\left\|v_{n}\right\|^{2}}{T^{2}}\right)\right|\|u\|^{6} \leq 16 T^{6} .
$$

(b) If $8 \lambda T^{2} \leq a$, then $a-4 \lambda T^{2} \geq \frac{a}{2}$ and estimate (3.23) implies that for every $u \in$ $H_{0}^{1}\left(R^{+}\right)$, one has

$$
\left|a+\lambda h_{T}(u)\|u\|^{2}+\frac{\lambda}{2 T^{2}} \psi^{\prime}\left(\frac{\|u\|^{2}}{T^{2}}\right)\|u\|^{4}\right| \geq \frac{a}{2} .
$$

Using the above lemmas, from Theorem 2.2 we obtain the following result.

Lemma 3.6. Assume that $4 \lambda T^{2}<a$. For almost every $\mu \in I$, there exists $v^{\mu} \in H_{0}^{1}\left(\mathbb{R}^{+}\right) \backslash\{0\}$ such that

$$
\left(J_{\lambda, \mu}^{T}\right)^{\prime}\left(v^{\mu}\right)=0 \quad \text { and } \quad J_{\lambda, \mu}^{T}\left(v^{\mu}\right)=c_{\lambda, \mu} .
$$

Proof. The above lemmas guarantee that for each $\lambda \geq 0$, Theorem 2.2 applies to the family of functionals $\left\{J_{\lambda, \mu}^{T}\right\}$. Hence, for almost every $\mu \in I$, we can find a bounded sequence $\left\{v_{n}^{\mu}\right\} \subset H_{0}^{1}\left(\mathbb{R}^{+}\right)$satisfying

$$
J_{\lambda, \mu}^{T}\left(v_{n}^{\mu}\right) \rightarrow c_{\lambda, \mu} \text { and }\left(J_{\lambda, \mu}^{T}\right)^{\prime}\left(v_{n}^{\mu}\right) \rightarrow 0 \text { as } n \rightarrow+\infty .
$$

Now, if $4 \lambda T^{2}<a$, then according to Lemma 3.5, there exists $v^{\mu} \in H_{0}^{1}\left(\mathbb{R}^{+}\right)$such that up to a subsequence $v_{n}^{\mu} \rightarrow v^{\mu}$ in $H_{0}^{1}\left(\mathbb{R}^{+}\right)$. So (3.25) and the continuity of $J_{\lambda, \mu}^{T}$ and $\left(J_{\lambda, \mu}^{T}\right)^{\prime}$ implies (3.24). Finally, from $c_{\lambda, \mu} \geq c>0$ it follows that $v^{\mu} \neq 0$.

Our next result is a Pohozaev type identity.

Lemma 3.7. If $u \in H_{0}^{1}\left(\mathbb{R}^{+}\right)$is a critical point of $J_{\lambda, \mu}^{T}$, i.e., $u$ is a weak solution of the problem (3.26)

$$
\left\{\begin{array}{l}
\left(a+\lambda h_{T}(u)\|u\|^{2}+\frac{\lambda}{2 T^{2}} \psi^{\prime}\left(\frac{\|u\|^{2}}{T^{2}}\right)\|u\|^{4}\right)\left(-u^{\prime \prime}(t)+b u(t)\right)=\mu \widetilde{f}(u(t)) \text { for a.e. } t \in(0,+\infty), \\
u(0)=u(+\infty)=0,
\end{array}\right.
$$


then

$$
\begin{aligned}
& \left(\frac{1}{2} \int_{0}^{+\infty}\left(-u^{\prime}(t)^{2}+b u(t)^{2}\right) \mathrm{d} t\right)\left(a+\lambda h_{T}(u)\|u\|^{2}+\frac{\lambda}{2 T^{2}} \psi^{\prime}\left(\frac{\|u\|^{2}}{T^{2}}\right)\|u\|^{4}\right) \\
= & \mu \int_{0}^{+\infty} \widetilde{F}(u(t)) \mathrm{d} t .
\end{aligned}
$$

Proof. Let $\mathcal{S}(s): H_{0}^{1}\left(\mathbb{R}^{+}\right) \longrightarrow H_{0}^{1}\left(\mathbb{R}^{+}\right)$be the family of transformations $\mathcal{S}(s) u(t)=u\left(\frac{t}{s}\right)$, $s>0$. Clearly, for any $u \in H_{0}^{1}\left(\mathbb{R}^{+}\right)$one has

$$
\begin{aligned}
J_{\lambda, \mu}^{T}(\mathcal{S}(s) u)= & \frac{a}{2}\left(\int_{0}^{+\infty}\left(\frac{1}{s} u^{\prime}(t)^{2}+b s u(t)^{2}\right) \mathrm{d} t\right)+\frac{\lambda}{4} \psi\left(\frac{\int_{0}^{+\infty}\left(\frac{1}{s} u^{\prime}(t)^{2}+b s u(t)^{2}\right) \mathrm{d} t}{T^{2}}\right) \\
& \times\left(\int_{0}^{+\infty}\left(\frac{1}{s} u^{\prime}(t)^{2}+b s u(t)^{2}\right) \mathrm{d} t\right)^{2}-\mu \int_{0}^{+\infty} s \widetilde{F}(u(t)) \mathrm{d} t .
\end{aligned}
$$

If $u$ is a critical point of $J_{\lambda, \mu}^{T}$, then since $S(1)=\mathrm{id}$, we have

which by (3.28) gives

$$
\left.\frac{d}{d s} J_{\lambda, \mu}^{T}(\mathcal{S}(s) u)\right|_{s=1}=0
$$

$$
\begin{gathered}
\frac{a}{2}\left(\int_{0}^{+\infty}\left(-u^{\prime}(t)^{2}+b u(t)^{2}\right) \mathrm{d} t\right)+\frac{\lambda}{4 T^{2}}\left(\int_{0}^{+\infty}\left(-u^{\prime}(t)^{2}+b u(t)^{2}\right) \mathrm{d} t\right) \psi^{\prime}\left(\frac{\|u\|^{2}}{T^{2}}\right)\|u\|^{4} \\
+\frac{\lambda}{2}\left(\int_{0}^{+\infty}\left(-u^{\prime}(t)^{2}+b u(t)^{2}\right) \mathrm{d} t\right) \psi\left(\frac{\|u\|^{2}}{T^{2}}\right)\|u\|^{2}-\mu \int_{0}^{+\infty} \widetilde{F}(u(t)) \mathrm{d} t=0,
\end{gathered}
$$

that is (3.27).

The next lemma will help us to come back to the untruncated functional $J_{\lambda}$.

Lemma 3.8. For $T>0$ sufficiently large, there exists $\lambda_{0}>0$ such that for every $\lambda \in\left[0, \lambda_{0}\right)$ and $\mu \in I$, any critical point $u$ of the functional $J_{\lambda, \mu}^{T}$ at level $c_{\lambda, \mu}$ is such that $\|u\| \leq T$.

Proof. Let $u$ be any critical point of $J_{\lambda, \mu_{n}}^{T}$ at level $c_{\lambda, \mu}$. From Lemma 3.7 we have

$$
\mu \int_{0}^{+\infty} \widetilde{F}(u(t)) \mathrm{d} t=\left(\frac{1}{2} i n t_{0}^{+\infty}\left(-u^{\prime}(t)^{2}+b u(t)^{2}\right) \mathrm{d} t\right) \times\left(a+\lambda h_{T}(u)\|u\|^{2}+\frac{\lambda}{2 T^{2}} \psi^{\prime}\left(\frac{\|u\|^{2}}{T^{2}}\right)\|u\|^{4}\right)
$$

This combined with $J_{\lambda, \mu}^{T}(u)=c_{\lambda, \mu}$ yields

$$
\begin{aligned}
& \left(\int_{0}^{+\infty} u^{\prime}(t)^{2} \mathrm{~d} t\right) \times\left(a+\lambda h_{T}(u)\|u\|^{2}+\frac{\lambda}{2 T^{2}} \psi^{\prime}\left(\frac{\|u\|^{2}}{T^{2}}\right)\|u\|^{4}\right) \\
= & c_{\lambda, \mu}+\frac{\lambda}{4} h_{T}(u)\|u\|^{4}+\frac{\lambda}{4 T^{2}} \psi^{\prime}\left(\frac{\|u\|^{2}}{T^{2}}\right)\|u\|^{6} .
\end{aligned}
$$

Now from Remark 3.1 (a) we have

$$
0 \leq \frac{\lambda}{4} h_{T}(u)\|u\|^{4} \leq \lambda T^{4} \text { and } \quad \frac{\lambda}{4 T^{2}}\left|\psi^{\prime}\left(\frac{\|u\|^{2}}{T^{2}}\right)\right|\|u\|^{6} \leq 4 \lambda T^{4},
$$

which if $8 \lambda T^{2} \leq a$, in view of Remark $3.1(\mathrm{~b})$ implies

$$
\begin{aligned}
\frac{a}{2} \int_{0}^{+\infty} u^{\prime}(t)^{2} \mathrm{~d} t & \leq\left|a+\lambda h_{T}(u)\|u\|^{2}+\frac{\lambda}{2 T^{2}} \psi^{\prime}\left(\frac{\|u\|^{2}}{T^{2}}\right)\|u\|^{4}\right|\left(\int_{0}^{+\infty} u^{\prime}(t)^{2} \mathrm{~d} t\right) \\
& =\left|c_{\lambda, \mu}+\frac{\lambda}{4} h_{T}(u)\|u\|^{4}+\frac{\lambda}{4 T^{2}} \psi^{\prime}\left(\frac{\|u\|^{2}}{T^{2}}\right)\|u\|^{6}\right| \leq c_{\lambda, \mu}+5 \lambda T^{4} .
\end{aligned}
$$


Next we also need to estimate $c_{\lambda, \mu}$. From the definition of $c_{\lambda, \mu}$ and the proof of Lemma 3.3, we have

$$
\begin{aligned}
c_{\lambda, \mu} & \leq \max _{s \geq 0} J_{\lambda, \mu}^{T}(s \phi) \\
& \leq \max _{s \geq 0}\left\{\frac{a}{2} s^{2}+\frac{\lambda}{4} \psi\left(\frac{s^{2}}{T^{2}}\right) s^{4}-\mu \int_{0}^{+\infty} \widetilde{F}(s \phi) \mathrm{d} t\right\} \\
& =\max _{s \geq 0}\left\{\frac{a}{2} s^{2}-\mu \int_{0}^{+\infty} \widetilde{F}(s \phi) \mathrm{d} t\right\}+\max _{s \geq 0}\left\{\frac{\lambda}{4} \psi\left(\frac{s^{2}}{T^{2}}\right) s^{4}\right\} \\
& \leq \max _{s \geq 0}\left\{\left(\frac{a}{2}-M \delta \int_{0}^{R} \phi^{2}(t) \mathrm{d} t\right) s^{2}+C(M) R\right\}+\lambda T^{4} \\
& =C(M) R+\lambda T^{4} .
\end{aligned}
$$

Hence (3.31), yields

$$
\frac{a}{2} \int_{0}^{+\infty} u^{\prime}(t)^{2} \mathrm{~d} t \leq C(M) R+6 \lambda T^{4}
$$

By using (3.14), (2.5) and the fact that $\left(J_{\lambda, \mu}^{T}\right)^{\prime}(u)=0$, we obtain

$$
\begin{aligned}
& a\|u\|^{2}+\lambda h_{T}(u)\|u\|^{4}+\frac{\lambda}{2 T^{2}} \psi^{\prime}\left(\frac{\|u\|^{2}}{T^{2}}\right)\|u\|^{6} \\
= & \mu \int_{0}^{+\infty} \widetilde{f}\left(u_{n}(t)\right) u_{n}(t) \mathrm{d} t \leq \int_{0}^{+\infty}\left(\epsilon|u(t)|+C_{\epsilon}|u(t)|^{\theta}\right)|u(t)| \mathrm{d} t \\
= & \epsilon \int_{0}^{+\infty} u(t)^{2} \mathrm{~d} t+C_{\epsilon} \int_{0}^{+\infty}|u(t)|^{\theta+1} \mathrm{~d} t \leq \epsilon\|u\|_{2}^{2}+C_{\epsilon} \int_{0}^{+\infty} \frac{1}{p(t)^{\theta+1}} p^{\theta+1}(t)|u(t)|^{\theta+1} \mathrm{~d} t \\
\leq & \frac{\epsilon}{b}\|u\|^{2}+C_{\epsilon}\left\|p^{-(\theta+1)}\right\|_{1}\|u\|_{\infty, p}^{\theta+1} \\
\leq & \frac{\epsilon}{b}\|u\|^{2}+C_{\epsilon} c_{\infty, p}^{\theta+1}\left\|p^{-(\theta+1)}\right\|_{1}\left(\int_{0}^{+\infty} u^{\prime}(t)^{2} \mathrm{~d} t\right)^{(\theta+1) / 2} .
\end{aligned}
$$

Hence by (3.33) we obtain

$$
\begin{aligned}
\left(a-\frac{\epsilon}{b}\right)\|u\|^{2} & \leq C_{\epsilon} c_{\infty, p}^{\theta+1}\left\|p^{-(\theta+1)}\right\|_{1}\left(\int_{0}^{+\infty} u^{\prime}(t)^{2} \mathrm{~d} t\right)^{(\theta+1) / 2}-\frac{\lambda}{2 T^{2}} \psi^{\prime}\left(\frac{\|u\|^{2}}{T^{2}}\right)\|u\|^{6} \\
& \leq C_{\epsilon} c_{\infty, p}^{\theta+1}\left\|p^{-(\theta+1)}\right\|_{1}\left(\frac{2}{a}\right)^{(\theta+1) / 2}\left(C(M) R+6 \lambda T^{4}\right)^{(\theta+1) / 2}+8 \lambda T^{4} .
\end{aligned}
$$

Now choose $T>0$ large enough that

$$
\left(a-\frac{\epsilon}{b}\right) T^{2}>C_{\epsilon} c_{\infty, p}^{\theta+1}\left\|p^{-(\theta+1)}\right\|_{1}\left(\frac{2}{a}\right)^{(\theta+1) / 2}(C(M) R)^{(\theta+1) / 2} .
$$

Then there exists $\lambda_{0}>0$ small enough such that for any $\lambda \in\left[0, \lambda_{0}\right)$, the following inequality holds

$$
\left(a-\frac{\epsilon}{b}\right) T^{2} \geq C_{\epsilon} c_{\infty, p}^{\theta+1}\left\|p^{-(\theta+1)}\right\|_{1}\left(\frac{2}{a}\right)^{(\theta+1) / 2}\left(C(M) R+6 \lambda T^{4}\right)^{(\theta+1) / 2}+8 \lambda T^{4} .
$$

Clearly, from (3.34) and (3.35) we have $\|u\| \leq T$ as we wished. 
Proof of Theorem 1.1. Let $T$ and $\lambda_{0}$ be fixed according to Lemma 3.8. From Lemma 3.6, there is a sequence $\left\{\mu_{n}\right\} \subset I$ with $\mu_{n} \rightarrow 1^{-}$such that for each $n \geq 1$, functional $J_{\lambda, \mu_{n}}^{T}$ has a critical point $u_{n}$ at level $c_{\lambda, \mu_{n}}$, i.e., $u_{n} \in H_{0}^{1}\left(\mathbb{R}^{+}\right) \backslash\{0\}$ and

$$
J_{\lambda, \mu_{n}}^{T}\left(u_{n}\right)=c_{\lambda, \mu_{n}}, \quad\left(J_{\lambda, \mu_{n}}^{T}\right)^{\prime}\left(u_{n}\right)=0 .
$$

By Lemma 3.8 and inequality (3.32), one has $\left\|u_{n}\right\| \leq T$ for every $n$ and the sequence $\left\{c_{\lambda, \mu_{n}}\right\}$ is bounded. Then $h_{T}\left(u_{n}\right)=1$ and

$$
J_{\lambda, \mu_{n}}^{T}\left(u_{n}\right)=\frac{a}{2}\left\|u_{n}\right\|^{2}+\frac{\lambda}{4}\left\|u_{n}\right\|^{4}-\mu_{n} \int_{0}^{+\infty} \widetilde{F}\left(u_{n}(t)\right) \mathrm{d} t .
$$

We claim that $\left\{u_{n}\right\}$ is a Palais-Smale sequence of $J_{\lambda}$. Indeed, by using (3.14) and (3.15) one has

$$
\begin{gathered}
\int_{0}^{+\infty} \widetilde{f}\left(u_{n}(t)\right) u_{n}(t) \mathrm{d} t \leq \frac{\epsilon}{b}\left\|u_{n}\right\|^{2}+C_{\epsilon} c_{\infty, p}^{\theta+1}\left\|p^{-(\theta+1)}\right\|_{1}\left\|u_{n}\right\|^{\theta+1}, \\
\left.\int_{0}^{+\infty} \widetilde{F}\left(u_{n}(t)\right) \mathrm{d} t \leq \frac{a}{2}\left\|u_{n}\right\|^{2}+\frac{\epsilon}{2 b}\left\|u_{n}\right\|^{2}+\frac{C_{\epsilon}}{\theta+1} c_{\infty, p}^{\theta+1}\left\|p^{-(\theta+1)}\right\|_{1}\right)\left\|u_{n}\right\|^{\theta+1} .
\end{gathered}
$$

Furthermore, we have

$$
\begin{gathered}
J_{\lambda}\left(u_{n}\right)=J_{\lambda, \mu_{n}}^{T}\left(u_{n}\right)+\left(\mu_{n}-1\right) \int_{0}^{+\infty} \widetilde{F}\left(u_{n}(t)\right) \mathrm{d} t=c_{\lambda, \mu_{n}}+\left(\mu_{n}-1\right) \int_{0}^{+\infty} \widetilde{F}\left(u_{n}(t)\right) \mathrm{d} t, \\
\left\langle J_{\lambda}^{\prime}\left(u_{n}\right), v\right\rangle=\left\langle\left(J_{\lambda, \mu_{n}}^{T}\right)^{\prime}\left(u_{n}\right), v\right\rangle+\left(\mu_{n}-1\right) \int_{0}^{+\infty} \widetilde{f}\left(u_{n}(t)\right) v(t) \mathrm{d} t=\left(\mu_{n}-1\right) \int_{0}^{+\infty} \widetilde{f}\left(u_{n}(t)\right) v(t) \mathrm{d} t .
\end{gathered}
$$

From (3.36) and (3.37), the boundedness of $\left\{u_{n}\right\}$ and $c_{\lambda, \mu_{n}}$, imply that $\int_{0}^{+\infty} \tilde{f}\left(u_{n}(t)\right) v(t) \mathrm{d} t$ and $\int_{0}^{+\infty} \widetilde{F}\left(u_{n}(t)\right) \mathrm{d} t$ are bounded. Thus, when $\mu_{n} \rightarrow 1$, we have that $\left\{J_{\lambda}\left(u_{n}\right)\right\}$ is bounded and $J_{\lambda}^{\prime}\left(u_{n}\right) \rightarrow 0$. Therefore $\left\{u_{n}\right\}$ is a bounded Palais-Smale sequence of $J_{\lambda}$. Since for $\|u\| \leq T$ one has $J_{\lambda}(u)=J_{\lambda, 1}^{T}(u)$ and $J_{\lambda}^{\prime}(u)=\left(J_{\lambda, 1}^{T}\right)^{\prime}(u)$, Lemma 3.5 applies and guarantees that $\left\{u_{n}\right\}$ has a convergent subsequence. Thus we may assume that $u_{n} \rightarrow u$ in $H_{0}^{1}\left(\mathbb{R}^{+}\right)$. Then $J_{\lambda}^{\prime}(u)=0$ and according to Lemma 3.4, we have that

$$
J_{\lambda}(u)=\lim _{n \rightarrow+\infty} J_{\lambda}\left(u_{n}\right)=\lim _{n \rightarrow+\infty} J_{\lambda, \mu_{n}}^{T}\left(u_{n}\right)=\lim _{n \rightarrow+\infty} c_{\lambda, \mu_{n}} \geq c>0 .
$$

Consequently, $u$ is a nontrivial solution of (2.11) and $|u|$ is a nontrivial nonnegative solution of (1.1). The proof is thus complete.

Now we present two examples of nonlinearity $f$ which satisfies the hypotheses $(H 1)-(H 3)$ but not the Ambrosetti-Rabinowitz condition (AR). Note that, since the term $\frac{\lambda}{4}\|u\|^{4}$ in the expression of the functional $J_{\lambda}$ associated to our problem (1.1) is homogeneous of degree 4 , the corresponding $(A R)$ condition on $f$ is the following 4-superlinear growth condition $(A R)$ : there existe $\nu>4$ and $R>0$, such that

$$
0<\nu F(t) \leq t f(t), \quad \text { for all } \quad t>R .
$$

Example 3.1. Let $f: \mathbb{R}^{+} \rightarrow \mathbb{R}^{+}$be defined by $f(t)=4 t^{3} \arctan (t)+\frac{t^{4}}{1+t^{2}}$. A simple computation shows that $F(t)=t^{4} \arctan (t)$. It is easy to check that $f$ satisfies all the conditions (H1)-(H3) of Theorem 1.1, but not the (AR)-condition, because

$$
\begin{aligned}
t f(t)-\nu F & =(4-\nu) t^{4} \arctan (t)+\frac{t^{5}}{1+t^{2}}-\nu t^{4} \arctan (t) \\
& =t^{4}\left[(4-\nu) \arctan (t)+\frac{t}{1+t^{2}}\right] \rightarrow-\infty, \quad \text { as } t \rightarrow+\infty
\end{aligned}
$$


for any $\nu>4$.

Example 3.2. An other example is given by the function $f(t)=4 t^{3} \ln (1+t)+\frac{t^{4}}{1+t}$, for $t \in[0,+\infty)$, with $F(t)=t^{4} \ln (t+1)$.

\section{REFERENCES}

[1] Bonanno, G. and O'Regan, D., A boundary value problem on the half-line via critical point methods, Dynam. Systems Appl., 15 (2006), 395-408

[2] Boulaiki, H., Moussaoui, T. and Precup, R., Multiple positive solutions for a second-order boundary value problem on the half-line, J. Nonlinear Funct. Anal., 2017 (2017), 1-25

[3] Brézis, H., Functional analysis, Sobolev spaces and partial differential equations, Springer, New York, 2010

[4] Caristi, G., Heidarkhani, S. and Salari, A., Variational approaches to Kirchhoff-type second-order impulsive differential equations on the half-line, Results Math., 73 (2018), No. 1, Paper No. 44, 31 pp.

[5] Dickey, R. W., The initial value problem for a non linear semi-infinite string, Proc. Roy. Soc. Edinburgh Sect. A, $82(1978), 19-26$

[6] Duan, Y. and Zhou, Y., Existence of solutions for Kirchhoff type equations with unbounded potential, Electron. J. Differential Equations, 2017 (2017), 184, 1-12

[7] Gomes, J. M. and Sanchez, L., A variational approach to some boundary value problems in the half-line, Z. Angew. Math. Phys., 56 (2005), 192-209

[8] Greenberg, J. M. and Hu, S. C., The initial-value problem for a stretched string, Quart. Appl. Math., 38 (1980), 289-311

[9] Heidarkhani, S., Afrouzi, G. A. and Moradi, S., Existence results for a Kirchhoff type second-order differential equation on the half-line with impulses, Asymptot. Anal., 105 (2017), 137-158

[10] Jeanjean, L., On the existence of bounded Palais-Smale sequences and application to a Landsman-Lazer-type problem set on $\mathbb{R}^{N}$, Proc. Roy. Soc. Edinburgh Sect. A, 129 (1999), 787-809

[11] Jeanjean, L., Local condition insuring bifurcation from the continuous spectrum, Math. Z., 232 (1999), 651-664

[12] Jeanjean, L. and Le Coz, S., An existence stability result for standing waves of nonlinear Schrödinger equations, Adv. Differential Equations,11 (2006), 813-840

[13] Kikuchi, H., Existence and stability of standing saves for Schrodinger-Poisson-Slater equation, Adv. Nonlinear Stud., 7 (2007), 403-437

[14] Kirchhoff, G., Vorlesungen Uber Mechanik, Teubner, Leipzig, 1883

[15] Li, Y., Li, F. and Shi, J., Existence of positive solutions to Kirchhoff type problems without compactness conditions, J. Differential Equations, 253 (2012), 2285-2294

[16] Li, Y., Li, F. and Shi, J., Existence of positive solutions to Kirchhoff type problems with zero mass, J. Math. Anal. Appl., 410 (2014), 361-374

[17] Ma, R. and B. Zhu, B., Existence of positive solutions for a semipositone boundary value problem on the half-line, Comput. Math. Appl., 58 (2009), 1672-1686

[18] Perera, A. K. and Zhang, Z., Nontrivial solutions of Kirchhoff-type problems via the Yang index, J. Differential Equations, 221 (2006), 246-255

[19] Struwe, M., Variational Methods, Springer, New York, 1996

[20] Zima, M., On positive solution of boundary value problems on the half-line, J. Math. Anal. Appl., 259 (2001), 127-136

FACULTY OF MATHEMATICS

USTHB, Po. Box 32, El-Alia Bab-ezzouar Algiers, Algeria

E-mail address: habiba_boulaiki@yahoo.fr

LABORATORY OF FIXED POINT THEORY AND APPLICATIONS

École Normale Supérieure, Kouba, Algiers, Algeria

E-mail address: toufik.moussaoui@gmail.com

DEPARTMENT OF MATHEMATICS

BABEŞ-BOlyai UNIVERSITY, ClUJ-NAPOCA, ROMANIA

E-mail address: r.precup@math. ubbcluj.ro 